\title{
Introduction to special issue on information privacy and trust in social media
}

\author{
Heng $\mathrm{Xu}^{1, *}$, Chuan-Hoo Tan ${ }^{2}$ \\ ${ }^{1}$ College of Information Sciences and Technology, Pennsylvania State University, University Park, PA 16802, USA; \\ ${ }^{2}$ College of Business, City University of Hong Kong, Tat Chee Avenue, Kowloon Tong, Hong Kong
}

Received on 7 June 2011

Copyright (C) $2011 \mathrm{Xu}$ and Tan, licensed to ICST. This is an open access article distributed under the terms of the Creative Commons Attribution licence (http://creativecommons.org/licences/by/3.0/), which permits unlimited use, distribution and reproduction in any medium so long as the original work is properly cited.

doi: $10.4108 /$ trans.sesa.2011.e2

The Web 2.0 indicates an emerging 'social' approach to generating and distributing Web content, characterized by open communication, decentralization of authority, and freedom to share and reuse [1]. The formation of dynamic coalitions aiming to share services and data can benefit from semantic web applications to facilitate cooperation across entities and users using different technologies. In the literature, these technologies have been referred to as social computing, which is a set of 'applications and services that facilitate collective action and social interaction online with rich exchange of multimedia information and evolution of aggregate knowledge' [1, p. 762].

In the platform of social computing, marked by active user participations and user-generated contents, privacy and security issues become particularly salient because a larger volume of user digital footprints could be potentially accessible to the public. As one PEW survey pointed out, the vast array of data constituting 'personal information' in the social computing platforms is nearly impossible to quantify or neatly define [2]. Users often act in such a way that the applications can observe and record, the potential knowledge in that action can be released onto the web, and made available to everyone. Social computing brought the voluntary disclosure of personal information to the mainstream, thus exposing users' published personal information to potential abuse by online crooks, stalkers, bullies, and even peers.

The goal of this special issue is to report frontier research addressing the need for a paradigm shift in understanding and addressing users' privacy and trust issues in social media. The wide varieties of methods used by studies published in this special issue are derived from computer science, human-computer interaction, informatics, statistics, and social sciences. The special issue aims to offer an integrated view of the field by presenting approaches originating from and drawing upon multiple disciplines.

There are three papers in this special issue. In the first paper authored by Motahari, Mayer, and Jones, the authors examine the problem of social inference and how it is associated to an individual's privacy risk. This is an excellent piece of work on social inference, which could stimulate significant endeavour on this specific area of research. In the second paper, Razavi and Gillet present a framework for developing privacy management mechanisms for social software systems. Developing from the grounded theory approach, the authors exemplify through the design of OpnTag prototype that personal and social information management and sharing could be performed. The last paper is co-authored by $\mathrm{Xu}$, Dinev, and $\mathrm{Li}$. This empirical study proposes and validates a research model that postulates trust beliefs and perceived risks as two major antecedent beliefs to the usage intention in the context of peer-to-peer file sharing.

Overall, this special issue illustrates the major challenges in examining the interplay between social and technological issues associated with security, privacy, and trust in the platform of social computing, as well as future perspectives on privacy and security research in the information age. We hope that these articles will inspire collaboration among various disciplines and stimulate new research in this area.

\section{References}

[1] Parameswaran, M. and Whinston, B.A. (2007) Social computing: an overview. Commun. Assoc. Inf. Syst. 19: 762-780.

[2] Madden, M., Fox, S., Smith, A. and Vitak, J. (2007) Digital Footprints: Online identity management and search in the age of transparency. PEW Internet \& American Life Project: http://pewresearch.org/pubs/663/digital-footprints. 